\title{
Neutrino probe of cosmic ray astrophysics and new physics at sub-fermi distances
}

\author{
Luis A. Anchordoqui [for the Pierre Auger Collaboration] \\ Department of Physics, University of Wisconsin-Milwaukee, Milwaukee, WI 53201
}

\begin{abstract}
We show that if the evolution of cosmic ray sources follows that of active galactic nuclei, the upper limit on the diffuse flux of tau neutrinos from the Pierre Auger Observatory marginally constrains the proton fraction at the end of the energy spectrum. We also discuss prospects to uncover new physics leptophobic interactions using future Auger data.
\end{abstract}

Keywords: ultra-high energy cosmic rays, neutrinos

PACS: $98.70 . \mathrm{Sa}, 96.50 . \mathrm{sb}, 96.50 \mathrm{sd}, 13.15 .+\mathrm{g}$

The Pierre Auger Observatory is a major international effort aiming at high-statistics study of highest energy cosmic rays to uncover their origins and nature [1]. These cosmic rays are too rare to be directly detected, but the direction, energy, and to some extent the nature of the primary particle can be inferred from the cascade of secondary particles induced when the primary impinges on the upper atmosphere. These cascades, or air showers, have been studied in the past by measuring the nitrogen fluorescence they produce in the atmosphere, or by directly sampling shower particles at ground level. Auger is a "hybrid" detector, exploiting both of these methods by employing an array of water Čerenkov detectors overlooked by fluorescence telescopes; on clear, dark nights air showers are simultaneously observed by both types of detectors, facilitating powerful reconstruction methods and control of the systematic errors which have plagued cosmic ray experiments to date. Auger also provides a promising way of detecting ultra-high energy neutrinos by looking for deeply-developing, large zenith angle $\left(>60^{\circ}\right)$ or horizontal air showers. At these large angles, hadronic showers have traversed the equivalent of several times the depth of the vertical atmosphere and their electromagnetic component has extinguished far away from the detector. Only very high energy coreproduced muons survive past 2 equivalent vertical atmospheres. Therefore, the shape of a hadronic (background in this case) shower front is very flat and very prompt in time. In contrast, a neutrino shower appears pretty much as a "normal" shower. It is therefore straightforward to distinguish neutrino induced events from background hadronic showers. Moreover, because of full flavor mixing, tau neutrinos could be as abundant as other species and so very low $v_{\tau}$ fluxes could be detected very efficiently by Auger detectors by looking at the interaction in the Earth crust of quasi horizontal $v_{\tau}$ inducing a horizontal cascade at the detector.

The Pierre Auger Observatory has confirmed a strong steepening of the cosmic ray flux above $10^{10.6} \mathrm{GeV}$ [2, 3]. If such a steepening is due to cosmic ray interactions with the cosmic micorwave background, the cosmic ray arrival directions at the energies above the suppression are expected to correlate with the nearby matter distribution, which is quite inhomogeneous. Observation of these correlations would provide a 
doorway towards cosmic ray astronomy (provided that particle deflections in the intervening galactic and extragalactic magnetic fields are relatively small). Anisotropy clues have been already reported in the data: cosmic ray events above $\simeq 10^{10.8} \mathrm{GeV}$ seem to correlate over angular scales of less than $6^{\circ}$ with the directions of nearby (distance $<100 \mathrm{Mpc}$ ) active galactic nuclei [4], though the evidence for such anisotropy has not strengthened in a more recent analysis with larger statistics [5]. In addition, upper limits on the diffuse neutrino flux and the ultra-high energy cosmic ray photon fraction have been established [5]. All these observations strongly support the hypothesis that cosmic ray acceleration takes place in astrophysical objects. The measurements of the variation of the depth of shower maximum with energy, interpreted with current hadronic interaction models, favour a mixed cosmic ray (protons + nuclei) composition at energies above $10^{8.6} \mathrm{GeV}$ [6]. It is interesting to note, however, that uncertainties in the protonair cross-section at the high energies characteristic of air showers may be large enough to alter this conclusion [7]. In what follows we show that limits on neutrino fluxes provide complementary information to ascertain the cosmic ray nature.

It is helpful to envision the cosmic ray engines as machines where protons are accelerated and (possibly) permanently confined by the magnetic fields of the acceleration region. The production of neutrons and pions and subsequent decay produces neutrinos, gamma rays, and cosmic rays. If the neutrino-emitting source also produces high energy cosmic rays, then pion production must be the principal agent for the high energy cutoff on the proton spectrum. Conversely, since the protons must undergo sufficient acceleration, inelastic pion production needs to be small below the cutoff energy; consequently, the plasma must be optically thin. Since the interaction time for protons is greatly increased over that of neutrons because of magnetic confinement, the neutrons escape before interacting, and on decay give rise to the observed cosmic ray flux. The foregoing can be summarized as four conditions on the characteristic nucleon interaction time scale $\tau_{\text {int }}$; the neutron (pion, muon) decay lifetime $\tau_{n(\pi, \mu)}$; the characteristic cycle time of confinement $\tau_{\text {cycle }}$; the cooling time scale of charged particles $\tau_{\text {cool }}$; and the total proton confinement time $\tau_{\text {conf }}$ : $(i) \tau_{\text {int }} \gg \tau_{\text {cycle }} ;(i i) \tau_{n}>\tau_{\text {cycle }} ;(i i i) \tau_{\text {int }} \ll \tau_{\text {conf }}$; (iv) $\tau_{\pi, \mu}<\tau_{\text {cool. }}$. The first condition ensures that the protons attain sufficient energy. Conditions $(i)$ and $(i i)$ allow the neutrons to escape the source before decaying. Condition (iii) permits sufficient interaction to produce neutrons and neutrinos. Condition (iv) ensures that cooling processes of secondary pions, and muons have negligible influence on the energy distribution of the emitted neutrons and neutrinos.

Within this working hypothesis the relative number and energy of the neutrinos and neutrons depend only on kinematics, which implies approximate equipartition of the decaying pion's energy between the neutrinos and the electron, and the relative radiation density in the source. On average, each interaction will produce $\eta$ neutrinos per neutron with relative energy $\varepsilon$ per neutrino, i.e.

$$
\eta=\frac{\left\langle N_{v}\right\rangle}{\left\langle N_{n}\right\rangle} \quad \text { and } \quad \varepsilon=\frac{\left\langle E_{v}\right\rangle}{\left\langle E_{n}\right\rangle} .
$$

The neutrino emissivity of flavor $i$ is then given by:

$$
\frac{\Delta E_{v_{i}}}{N_{v_{i}}} \mathscr{L}_{v_{i}}\left(z, E_{v_{i}}\right)=\frac{\Delta E_{n}}{N_{n}} \mathscr{L}_{n}\left(z, E_{n}\right) .
$$


Assuming flavor universality as well as $\varepsilon \simeq E_{v} / E_{n} \simeq \Delta E_{v} / \Delta E_{n}$ and $\eta \simeq N_{\text {all } v} / N_{n}$ we arrive at the neutrino source luminosity (per co-moving volume):

$$
\mathscr{L}_{\text {all } v}\left(z, E_{v}\right) \simeq \frac{\eta}{\varepsilon} \mathscr{L}_{n}\left(z, E_{v} / \varepsilon\right)
$$

In the following, we will consider a (hypothetical) source, where pion production proceeds exclusively via resonant $p \gamma \rightarrow \Delta^{+}$scattering with fixed values $\eta=3$ and $\varepsilon=0.07$. Note, that the relation (3) derived for optically thin sources can be regarded as a lower limit on the neutrino luminosity as long as energy-loss processes in the source are negligible. Next, we translate this conservative expectation into an upper limit on the extragalactic proton fraction in ultra-high energy cosmic rays, exploiting the experimental upper limit on the diffuse flux of tau neutrinos [5].

For this procedure we introduce test functions of the neutron source luminosity of the form $\mathscr{L}_{n}^{\text {test }}(0, E)=\mathscr{L}_{0}\left(E / E_{\max }\right)^{-1} \exp \left(-E / E_{\max }\right)$, with an exponential energy cut-off $E_{\max }$ that we vary between $10^{8} \mathrm{GeV}$ and $10^{12} \mathrm{GeV}$ with a logarithmic stepsize of $\log _{10} E=0.25$. We adopt the usual concordance cosmology of a flat universe dominated by a cosmological constant with $\Omega_{\Lambda} \sim 0.7$, the rest being cold dark matter with $\Omega_{\mathrm{m}} \sim 0.3$. The Hubble parameter is given by $H^{2}(z)=H_{0}^{2}\left(\Omega_{\mathrm{m}}(1+z)^{3}+\Omega_{\Lambda}\right)$, normalized to its value today of $70 \mathrm{~km} \mathrm{~s}^{-1} \mathrm{Mpc}^{-1}$. The time-dependence of the redshift can be expressed via $\mathrm{d} z=-\mathrm{d} t(1+z) H$. The cosmological evolution of the source density per co-moving volume is parameterized as $\mathscr{L}_{i}(z, E)=\mathscr{H}(z) \mathscr{L}_{i}(0, E)$, where the source luminosity per co-moving volume is assumed to follow that of gamma ray bursts $\left[\mathscr{H}_{\mathrm{GRB}}(z)=(1+z)^{4.8}\right.$, for $z<1 ; \mathscr{H}_{\mathrm{GRB}}(z)=N_{1}(1+z)^{3.1}$, for $1<z<4$; $\mathscr{H}_{\mathrm{GRB}}(z)=N_{1} N_{4}(1+z)^{-0.1}$, for $z>4$; with $N_{1}=2^{3.7}$ and $\left.N_{4}=5^{3.2}\right]$ and that of active galactic nuclei $\left[\mathscr{H}_{\mathrm{AGN}}(z)=(1+z)^{5.0}\right.$, for $z<1.7 ; \mathscr{H}_{\mathrm{AGN}}(z)=N_{1.7}$, for $1.7<z<2.7$; $\mathscr{H}_{\text {AGN }}(z)=N_{1.7} N_{2.7}^{(2.7-z)}$, for $z>2.7$; with $N_{1.7}=2.7^{5}$ and $\left.N_{2.7}=10^{0.43}\right]$. Each neutron test luminosity is related to a neutrino luminosity by the ratio (3). After propagation we normalize the accumulated contribution of extra-galactic and cosmogenic neutrinos to the limit on ultra-high energy tau neutrinos from Auger. For details on the calculation see [8]. The results are shown in Fig. 1. For $\mathscr{H}_{\mathrm{AGN}}(z)$, the upper limit on the diffuse flux of tau neutrinos marginally constrains the proton fraction somewhat around $10^{10} \mathrm{GeV}$.

Cosmic neutrinos are also unique probes of new physics as their interactions are uncluttered by the strong and electromagnetic forces and can reach center-of-mass energies $\sqrt{s} \sim 245 \mathrm{TeV}$. However, rates for new physics processes are difficult to test since the flux of cosmic neutrinos is virtually unknown. It is possible to mitigate this by using multiple observables which allow one to decouple effects of the flux and cross section. In particular, possible deviations of the neutrino nucleon cross section due to new physics leptophobic interactions (e.g., production and decay of TeV-scale black holes) can easily be uncovered at Auger by combining information of the two different neutrino channels [9]. Namely, if an anomalously large quasi-horizontal deep shower rate is found, it may be ascribed to either an enhancement of the incoming neutrino flux, or an enhancement in the neutrino-nucleon cross section. However, these two possibilities may be distinguished by separately binning events which arrive at very small angles to the horizontal, the so-called tau Earth-skimming events. An enhanced flux will increase both quasi-horizontal and Earth-skimming event rates, whereas a large 

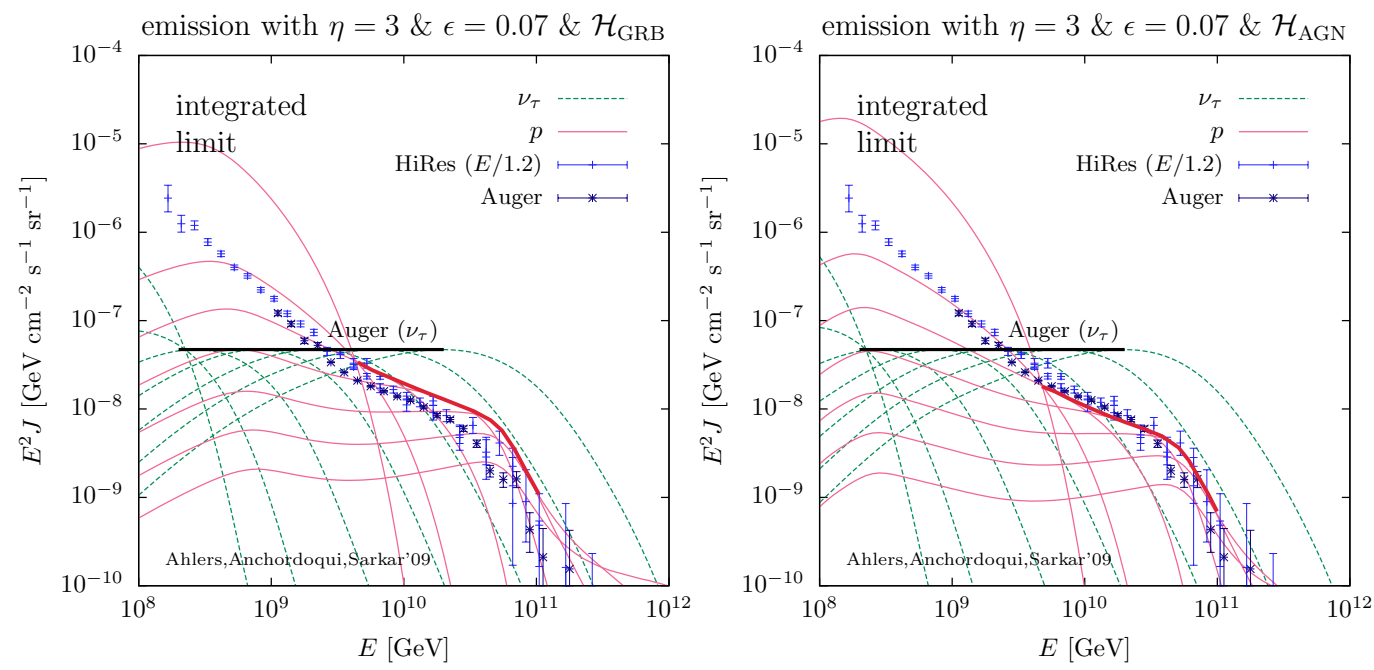

FIGURE 1. Upper limits on the proton contribution in ultra-high energy cosmic rays derived from Auger limit on the diffuse $v_{\tau}$-flux in models where the cosmic ray sources evolve strongly with redshift; we show the case of strong cosmological evolution of the proton sources according to $\mathscr{H}_{\mathrm{GRB}}$ and $\mathscr{H}_{\mathrm{AGN}}$.

black hole cross section suppresses the latter, because the hadronic decay products cannot escape the Earth crust. This argument requires only counting experiments and does not rely on measurements of shower properties.

In summary, statistics and better experimental handles should enable us to reconstruct the high end of the energy spectrum, to locate the cosmic ray sources in the sky, and to discern the primary mass composition. Future Auger data will not only provide clues to the cosmic ray origin, but could enhance our understanding of fundamental physics at sub-fermi distances. An optimist might even imagine the discovery of microscopic black holes, the telltale signature of the universe's unseen dimensions.

\section{ACKNOWLEDGMENTS}

I would like to thank Markus Ahlers, Jonathan Feng, Haim Goldberg, Subir Sarkar, and Al Shapere for the most enjoyable collaborations. This work has been partially supported by NSF Grant No PHY-0757598 and the UWM Research Growth Initiative.

\section{REFERENCES}

1. J. Abraham et al. [Pierre Auger Collaboration], Nucl. Instrum. Meth. A 523, 50 (2004).

2. J. Abraham et al. [Pierre Auger Collaboration], Phys. Rev. Lett. 101, 061101 (2008).

3. J. Abraham et al. [Pierre Auger Collaboration], arXiv:0906.2189.

4. J. Abraham et al. [Pierre Auger Collaboration], Science 318, 938 (2007).

5. J. Abraham et al. [Pierre Auger Collaboration], arXiv:0906.2347.

6. J. Abraham et al. [Pierre Auger Collaboration], arXiv:0906.2319.

7. R. Ulrich, R. Engel, S. Muller, F. Schussler and M. Unger, arXiv:0906.3075.

8. M. Ahlers, L. A. Anchordoqui and S. Sarkar, Phys. Rev. D 79, 083009 (2009).

9. L. A. Anchordoqui, J. L. Feng, H. Goldberg and A. D. Shapere, Phys. Rev. D 65, 124027 (2002). 